\section{An unusual cause of sudden dyspnoea}

A 68-year-old man was admitted as an emergency to a private hospital after becoming acutely dyspnoeic while out walking. Clinical examination and chest X-ray confirmed acute pulmonary oedema. Six weeks prior to admission he had undergone a bioprosthetic aortic valve replacement for severe aortic regurgitation. An urgent transthoracic echocardiogram revealed an aorto-left atrial communication. Subsequent transoesophageal echocardiogram (TOE) confirmed a fistulous communication between the aorta and left atrium (figure 1). The TOE images showed an aneurysmal dilatation in the annular region (figure 2) with blood tracking out of the aorta and swirling around the wall of the left atrium. There was no echocardiographic evidence of endocarditis or a paravalvular abscess. The images appeared to demonstrate dehiscence of the previously implanted bioprosthetic aortic valve with the creation of an aorto-left atrial fistulous communication resulting in the very acute presentation

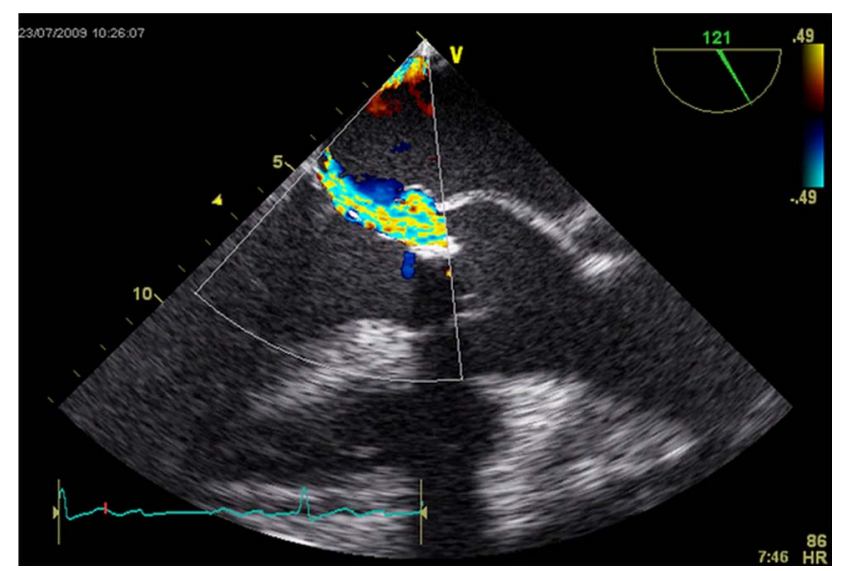

Figure 1 Transoesophageal echocardiogram showing aorto-left atrial communication. Blood can be seen tracking out of the aorta and swirling around the wall of the left atrium.

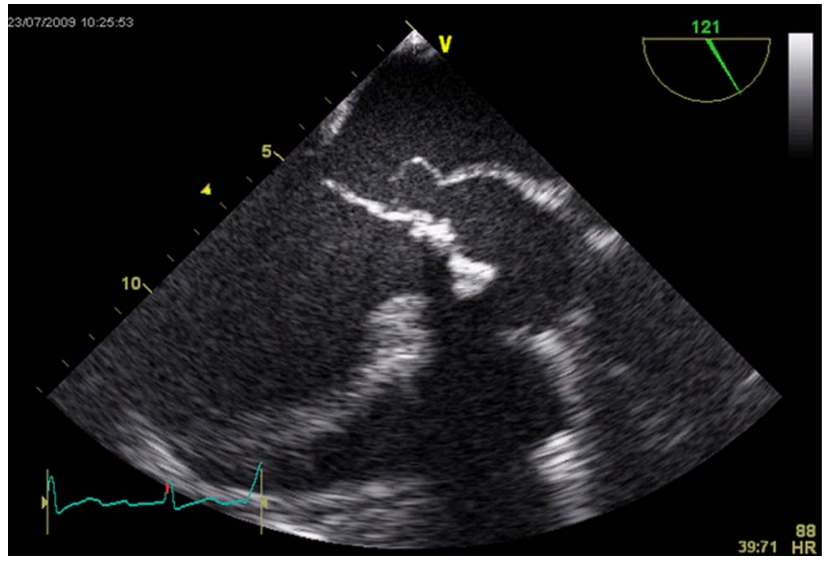

Figure 2 Transoesophageal echocardiogram showing the aneurysmal dilatation.

with pulmonary oedema. He was urgently transferred to the operating theatre and successfully underwent an aortic root replacement and mechanical aortic valve replacement. The images illustrate beautifully the aorto-atrial communication. Major valve dehiscence requires urgent attention and the report highlights the importance of prompt echocardiographic imaging and involvement of the cardiology and cardiothoracic teams in patients presenting with acute dyspnoea after having undergone recent valve replacement surgery.

\section{Tawfiq R Choudhury, ${ }^{1}$ Sundeep Puri ${ }^{2}$}

${ }^{1}$ Department of Cardiology, Manchester Royal Infirmary, Manchester, UK 2Department of Cardiology, Tameside General Hospital, Ashton-under-Lyne, UK

Correspondence to Dr Tawfiq R Choudhury, Department of Cardiology, Manchester Royal Infirmary, Oxford Road, Manchester M13 9WL, UK; tawfiqc@hotmail.com

Contributors TRC: gathering images, drafting article. SP: supervising consultant. Competing interests None.

Patient consent Obtained.

Provenance and peer review Not commissioned; internally peer reviewed. To cite Choudhury TR, Puri S. Heart Asia 2013;5:199.

Heart Asia 2013;5:199. doi:10.1136/heartasia-2013-010411 